\title{
DISTRIBUIÇÃO ESPACIAL DAS PROPRIEDADES DO SOLO, PRODUÇÃO DO CAPIM-MARANDU COM A INTENSIDADE DE PASTEJO
}

\author{
Nayara Martins Alencar ${ }^{1}$, Jonahtan Chaves Melo², Antonio Clementino dos Santos $^{3}$, Odislei Fagner Ribeiro Cunha², \\ Joaquim José de Paula Neto ${ }^{1}$
}

\section{RESUMO}

A existência de variabilidade espacial nas propriedades do solo influencia na produção da forrageira. O trabalho teve como objetivo determinar a distribuição espacial das propriedades químicas do solo relacionando-a com a produção e a preferência de forragem por animais em pastejo de Urochloa brizantha cv. Marandu (Syn. Brachiaria brizantha) sob lotação contínua. Para caracterização da área de pastagem, foram realizadas medições de 108 pontos com distribuição em malha irregular, com coletas de solo para determinação dos atributos químicos ( $\mathrm{pH}$, matéria orgânica, $\mathrm{P}, \mathrm{K}, \mathrm{Ca}, \mathrm{Mg}$, Al, H+Al) e densidade do solo, produção de forragem, altura do dossel forrageiro, índice SPAD e intensidade de pastejo, no período das águas de 2012. Realizou-se o teste de normalidade, seguido de análise descritiva dos dados e análise geoestatística. As características químicas e densidade do solo, bem como a produção e altura do pasto, apresentaram dependência espacial, com mesmo padrão de resposta, inferindo que tais respostas são interrelacionadas. Os fatores que causam a variabilidade nas características do solo também interferem nas relacionadas ao pasto influenciando em parte no pastejo animal. Os atributos químicos do solo, densidade, massa seca, altura e intensidade de pastejo, ajustaram-se ao modelo exponencial. A estimativa da variabilidade espacial dos atributos do solo juntamente com avaliação da disponibilidade do pasto e os pontos de preferência do pastejo animal são ferramentas importantes para estabelecer critérios de uso e manejo diferenciado dentro de uma mesma área de pastagem, na perspectiva de evitar degradação do solo e do pasto.

Palavras-chave: estrutura do pasto, geoestatística, krigagem, preferência de pastejo, Urochloa brizantha

\section{ABSTRACT \\ SPATIAL VARIABILITY OF SOIL PROPERTIES, PRODUCTION OF MARANDU-GRASSLAND AND INTERRELATION WITH THE GRAZING INTENSITY}

The existence of spatial variability in soil properties influences the production of forage. The study aimed to determine the spatial distribution of soil chemical properties relating it to the production and the preference of grazing animals in pasture Urochloa brizantha cv. Marandu (Syn. Brachiaria brizantha) under continuous stocking. To characterize the pasture area, were carried out measurements of 108 points with distribution in irregular mesh with samplings to determine the chemical properties ( $\mathrm{pH}$, organic matter, $\mathrm{P}, \mathrm{K}, \mathrm{Ca}, \mathrm{Mg}, \mathrm{Al}, \mathrm{H}+\mathrm{Al}$ ) and soil density , forage production, sward height, SPAD index and intensity of grazing in the water period of 2012. The normality test was made, followed by a descriptive analysis of the data analysis and geostatistics. Although the present condition of operation, it was observed that the chemical characteristics of the soil and soil density, as well as production and pasture height space dependence, presented same pattern of response, inferring that such responses are inter-relation. The factors that cause variability in soil characteristics, also interfere in influencing pasture related in part in grazing animal. Chemical soil attributes, density, dry mass, height, and grazing intensity, set the exponential model. The estimation of the spatial variability of soil attributes together with assessment of the availability of pasture, and animal grazing preference points, are important tools for establishing criteria for use and differentiated management within the same pasture area, in order to prevent degradation of the soil and grass.

Keywords: geostatistics, grazing preferably, kriging, sward structure, Urochloa brizantha

\section{Recebido para publicação em 10/05/2016. Aprovado em 08/08/2016.}

1 -Zootecnista, Doutorando em Ciência Animal Tropical, UFT/Araguaína-TO, nayara_m1@hotmail.com; jneto@zootecnista.com.br.

2-Zootecnista, Doutor em Ciência Animal Tropical, UFT/Araguaína-TO, jonahtancmelo@hotmail.com; ofrcunha@gmail.com.

3- Engenheiro Agrônomo, Professor Adjunto, UFT/Araguaína-TO, clementino@uft.edu.br

338 REVENG 338-349p. 


\section{INTRODUÇÃO}

A baixa produtividade no Ecótono CerradoAmazônia está relacionada à ausência na reposição dos nutrientes do solo, falta de ajuste da carga animal, uso do fogo e o mau manejo do solo e do pastejo. Dos 173 milhões de hectares de pastagens no Brasil, 117 milhões são de pastagens cultivadas e estima-se cerca de $70 \%$ das pastagens cultivadas encontra-se em algum estádio de degradação (MACEDO et al., 2013). Os sistemas intensivos de produção de bovinos a pasto, com base no aproveitamento da fertilidade natural, levam ao declínio da produtividade das pastagens, resultando na degradação do pasto (MARCHÃO et al., 2009). São necessárias práticas de manejo adequadas que busquem a manutenção da fertilidade dos solos e produtividade do pasto, com aplicação de corretivos, fertilizantes e a adequação do manejo do pasto, de acordo com espécie forrageira (SANA et al., 2014).

A escolha do local de pastejo pelos animais pode ser afetada pelo tamanho do pasto, topografia, posição das cercas, proximidade e facilidade de acesso a locais atrativos como aguada, cochos, sombra, além de locais com maior disponibilidade de forragem e nutrientes minerais disponíveis nas plantas (MELO et al., 2015; PÁSCOA e COSTA, 2007; TREVISAN et al., 2005), esses fatores influenciam na distribuição heterogênea das excretas no pasto (SILVA et al., 2013), bem como no aumento da não homogeneidade da área. Informações sobre o comportamento animal e sua localização dentro do pasto podem auxiliar para delimitar locais e ambientes a serem utilizados pelos animais em função das necessidades, bem como caracterizar a heterogeneidade local com distribuição de insumos de acordo com área, buscando a homogeneização da área e o equilíbrio na relação planta-animal (CARVALHO et al., 2009).

É evidente a carência de informações para compreensão integrada da relação entre as características químicas, físicas do solo, sua cobertura e as zonas que efetivamente os animais efetuam atividade de pastejo em ambientes pastoris heterogêneos. Por meio da geoestatística, é possível obter informações sobre relações espaciais entre as variáveis, com possibilidade de gerar um conjunto de informações práticas altamente aplicáveis às condições de campo. No entanto, Páscoa e Costa (2007) relataram que esta ferramenta é pouco utilizada, ao passo que este método de análise deixa inúmeras lacunas, pois o teste de média e regressão não retrata algumas das respostas biológicas que são de natureza espacial-temporal.

A existência de variabilidade espacial nas características químicas do solo pode determinar uma variação na massa de forragem e determinar locais de pastejo preferidos pelos animais. Objetivou-se determinar a distribuição espacial das propriedades químicas do solo relacionando-a com a produção e a preferência de forragem por animais em pastejo de Urochloa brizantha cv. Marandu (Syn. Brachiaria brizantha) sob lotação contínua.

\section{MATERIAL E MÉTODOS}

O trabalho foi realizado em área de pastagem na Fazenda Nossa Senhora da Aparecida, próximo a Araguaína-TO, no mês de abril de 2012, nas coordenadas geográficas aproximadas de 07007'39's e 4814'24”W. A vegetação da área em estudo é caracterizada como Ecótono CerradoAmazônia, com clima Aw, ou seja, tropical de verão úmido e período de estiagem no inverno, segundo a classificação de Köppen (1948), com temperatura média de $28^{\circ} \mathrm{C}$ e precipitação pluviométrica anual em torno de $1800 \mathrm{~mm}$. O solo da área é descrito como Argissolo Vermelho distroférrico (EMBRAPA, 2013).

As variáveis relacionadas com os atributos químicos do solo, cobertura vegetal, altura do dossel forrageiro, valor SPAD (Soil and Plant Analysis Development) foram avaliadas em 108 pontos de coleta amostrais por distribuição em malha irregular em uma área de 16,71 ha, de modo a representar as condições da área estudada. A área de estudo foi estabelecida há aproximadamente 20 anos, com pasto predominante de Urochloa brizantha cv. Marandu. As amostras de solo foram retiradas na profundidade de $0-10 \mathrm{~cm}$, acondicionadas em sacos plásticos e encaminhadas para o Laboratório de Solos-EMVZ/UFT, onde foram secas ao ar e peneiradas em malha de 2 $\mathrm{mm}$ e então obtidas amostras de terra fina seca 
ao ar (TFSA), para determinação do $\mathrm{pH}$, matéria orgânica, $\mathrm{P}, \mathrm{K}, \mathrm{Ca}, \mathrm{Mg}, \mathrm{Al}, \mathrm{H}+\mathrm{Al}$ (EMBRAPA, 2009). Com os resultados obtidos nas análises químicas do solo, foi calculada a soma de bases trocáveis $(\mathrm{SB})$, a capacidade de troca catiônica a $\mathrm{pH} 7\left(\mathrm{CTC}_{\mathrm{pH}}\right)$, a capacidade de troca catiônica efetiva $\left(\mathrm{CTC}_{\mathrm{e}}\right)$, a percentagem de saturação por bases (V\%), e a percentagem de saturação por Alumínio (m\%). Para determinação da densidade do solo foi utilizado o método do torrão parafinado (EMBRAPA, 1997).

Em cada ponto de coleta foram realizadas medições do índice SPAD, com base em duas leituras em três folhas (folha em expansão, primeira folha completamente expandida e última folha da base não senescente), obtendo-se a média de perfilho representativo da comunidade de perfilhos, com utilização de medidor portátil de clorofila SPAD CCM-200 (Opti-Sciences).

A determinação da média de altura do dossel forrageiro foi realizada com auxílio de régua graduada, foram medidos da base do perfilho até a linha de curvatura da última folha expandida em seis pontos distintos, próximo de cada ponto de coleta do solo. Considerou-se a altura média e a intensidade de pastejo para atribuir pontos de forma subjetiva em escala de um a três pontos, com: 1) área no ponto de coleta pouco pastejado, com altura média acima de $20 \mathrm{~cm}$, com folhas em expansão e completamente expandidas intactas; 2 ) área no ponto de coleta com folhas em expansão totalmente consumidas pode apresentar folhas completamente expandidas em algum grau de consumo, com altura média entre 10-20 cm; e 3) condição extrema em que tanto folhas em expansão quanto expandidas foram consumidas, com altura média inferior a $10 \mathrm{~cm}$.

A partir da altura média do pasto, realizou-se amostragem do capim Marandu, utilizando um quadro de amostragem de 1 x 0,6 $\mathrm{m}\left(0,6 \mathrm{~m}^{2}\right)$, onde toda a forragem contida em seu interior foi colhida rente ao solo e pesada para determinação da massa verde de forragem. Uma alíquota de cada ponto amostrado foi colocada em saco plástico, assim realizou-se homogeneização do material colhido e concomitantemente retirou-se outra alíquota geral, na qual foi realizado procedimento de secagem em estufa de ventilação de ar forçada à temperatura constante $55^{\circ} \mathrm{C}$ até atingir massa constante (SILVA e QUEIROZ, 2002), para determinação da massa seca total no interior do quadro de amostragem $(\mathrm{kg}$ $\mathrm{m}^{-2}$ ).

Para cada variável estudada realizou-se análise estatística descritiva, para observar as variações em torno da média aritmética por meio da mediana, variância, coeficientes de variação, assimetria, curtose e o tipo de distribuição. Foram submetidos ao teste de Kolmogorov-Smirnov (KS) a $5 \%$ de probabilidade, para testar hipótese de normalidade. A dependência espacial foi caracterizada por meio de análise geoestatística (VIEIRA, 2000), as observações e autocorrelações entre locais vizinhos foram calculadas por meio da semivariância $\widehat{Y}(\mathrm{~h})$, utilizando o software GS + (ROBERTSON, 2008), estimado pela seguinte equação:

$\hat{\gamma}(\mathrm{h})=\frac{1}{2 \mathrm{~N}(\mathrm{~h})} \sum_{\mathrm{i}=1}^{\mathrm{N}(\mathrm{h})}[\mathrm{Z}(\mathrm{Xi})-\mathrm{Z}(\mathrm{Xi}+\mathrm{h})]^{2}$

em que,

$\hat{\gamma}(h)=$ semivariância;

$\mathrm{N}(\mathrm{h})=$ número de pares de valores medidos [Z(xi), $\mathrm{Z}(\mathrm{xi}+\mathrm{h})]$, separados pela distância $\mathrm{h}$;

$\mathrm{Z}=$ valores das variáveis estudadas; $\mathrm{e}$

xi e $x i+h=$ valores definidos pela localização geográfica dos pontos de coleta no campo.

Os semivariogramas foram ajustados para definir os valores de efeito pepita $\left(\mathrm{C}_{0}\right)$, variância $\left(\mathrm{C}_{1}\right)$, patamar $\left(\mathrm{C}_{0}+\mathrm{C}_{1}\right)$ e alcance $(\mathrm{A})$. A seleção de modelos matemáticos e o ajuste dos semivariogramas foram realizados com base no maior grau de dependência espacial (GDE), menor soma do quadrado dos resíduos (SQR) e maior coeficiente de determinação $\left(R^{2}\right)$. A dependência espacial é dada pela equação:

$G D E=\left[\frac{c}{c 1+c 0}\right] 100$

em que,

GDE = grau de dependência espacial (\%);

$\mathrm{C}_{1}=$ variância; $\mathrm{e}$

$\mathrm{C}_{0}+\mathrm{C}=$ patamar.

O grau de dependência espacial foi classificado 
em: fraco para GDE $<25 \%$; moderado para GDE entre 25 e $75 \%$; e forte para GDE $>75 \%$ (ROBERTSON, 2008). A interpolação dos valores foi realizada pelo método geoestatístico de Krigagem, o que possibilitou a construção dos mapas de isolinhas das variáveis respostas por meio do software Surfer 8.0 (GOLDEN SOFTWARE, 2002).

\section{RESULTADOS E DISCUSSÃO}

As médias e medianas dos atributos químicos e densidade do solo, massa de forragem, leitura do valor SPAD, mensuração da altura do dossel forrageiro e intensidade do pastejo permaneceram próximas (Quadro 1). O que indica baixa assimetria, que pode ser confirmada por meio dos valores dos coeficientes de assimetria e curtose que demonstram que os parâmetros avaliados possuem distribuições assimétricas com valores próximos de zero, exceto os teores de $\mathrm{K}, \mathrm{Al} \mathrm{e} \mathrm{m} \%$, com coeficientes de assimetria positivos e altos com agrupamento dos valores acima do valor médio dessas variáveis.

Conforme proposta por Warrick e Nielsen (1980), os valores do coeficiente de variação (CV) das variáveis analisadas foram classificados entre baixa $(\mathrm{CV}<12 \%)$, média $(12<\mathrm{CV}<60 \%)$ e alta $(\mathrm{CV}>60 \%)$ variabilidade. Os atributos $\mathrm{pH}, \mathrm{P}, \mathrm{V} \% \mathrm{e}$ Ds apresentaram baixa variabilidade onde, segundo Sana et al. (2014), o pH do solo está relacionado com a disponibilidade de nutrientes, geralmente este atributo apresenta menor variabilidade espacial, torna-se desejável que o pH não esteja

Quadro 1. Estatística descritiva dos parâmetros avaliados em área com capim-Marandu em Argissolo Vermelho Distroférrico em Araguaína, TO (2015)

\begin{tabular}{|c|c|c|c|c|c|c|c|}
\hline \multirow{2}{*}{ Variável } & \multirow{2}{*}{ Média } & \multirow{2}{*}{ Mediana } & \multirow{2}{*}{ Variância } & \multicolumn{3}{|c|}{ Coeficientes } & \multirow{2}{*}{$\mathbf{K S}^{1}$} \\
\hline & & & & Variação (\%) & Assimetria & Curtose & \\
\hline $\mathrm{MO}\left(\mathrm{g} \mathrm{kg}^{-1}\right)$ & 16,57 & 16,40 & 15,22 & 23,54 & 0,60 & 0,54 & $0,07 * *$ \\
\hline $\mathrm{pH}\left(\mathrm{CaCl}_{2}\right)$ & 5,04 & 5,06 & 0,05 & 4,28 & 0,46 & 0,82 & $0,09 *$ \\
\hline $\mathrm{P}\left(\mathrm{mg} \mathrm{dm} \mathrm{dm}^{-3}\right)$ & 0,50 & 0,50 & 0,01 & 3,98 & 0,84 & 0,16 & $0,19 *$ \\
\hline $\mathrm{K}^{+}\left(\mathrm{cmol}_{\mathrm{c}} \mathrm{dm}^{-3}\right)$ & 0,16 & 0,13 & 0,01 & 69,00 & 1,34 & 0,94 & $0,26^{*}$ \\
\hline $\mathrm{Ca}^{2+}\left(\mathrm{cmol}_{\mathrm{c}} \mathrm{dm}^{-3}\right)$ & 4,23 & 4,07 & 1,71 & 30,91 & 0,36 & 0,01 & $0,07 * *$ \\
\hline $\mathrm{Mg}^{2+}\left(\mathrm{cmol}_{\mathrm{c}} \mathrm{dm}^{-3}\right)$ & 3,49 & 3,44 & 0,58 & 21,92 & 0,47 & $-0,14$ & $0,07 * *$ \\
\hline $\mathrm{Al}^{3+}\left(\mathrm{cmol}_{\mathrm{c}} \mathrm{dm}^{-3}\right)$ & 0,14 & 0,12 & 0,01 & 76,03 & 2,28 & 6,27 & $0,20 *$ \\
\hline $\mathrm{H}+\mathrm{Al}\left(\mathrm{cmol}_{\mathrm{c}} \mathrm{dm}^{-3}\right)$ & 6,15 & 6,08 & 0,93 & 15,67 & 0,96 & 2,36 & $0,08^{* *}$ \\
\hline $\mathrm{SB}\left(\mathrm{cmol}_{\mathrm{c}} \mathrm{dm}^{-3}\right)$ & 7,89 & 7,66 & 1,92 & 17,58 & 0,31 & $-0,44$ & $0,09 *$ \\
\hline $\mathrm{CTC}_{\mathrm{pH} 7}\left(\mathrm{cmol}_{\mathrm{c}} \mathrm{dm}^{-3}\right)$ & 13,88 & 13,77 & 2,80 & 12,06 & 0,56 & 0,49 & $0,08 * *$ \\
\hline $\mathrm{CTC}_{\mathrm{e}}\left(\mathrm{cmol}_{\mathrm{c}} \mathrm{dm}^{-3}\right)$ & 7,88 & 7,57 & 1,83 & 17,16 & 0,38 & $-0,33$ & $0,10^{*}$ \\
\hline V (\%) & 56,69 & 56,9 & 35,23 & 10,47 & 0,005 & $-0,03$ & $0,03 * *$ \\
\hline m (\%) & 1,94 & 1,56 & 2,59 & 82,92 & 2,64 & 8,47 & $0,18^{*}$ \\
\hline Ds $\left(\mathrm{kg} \mathrm{dm}^{-3}\right)$ & 1,16 & 1,15 & 0,01 & 10,83 & 0,19 & $-0,25$ & $0,07 * *$ \\
\hline $\operatorname{MST}\left(\mathrm{kg} \mathrm{m}^{-2}\right)$ & 0,52 & 0,53 & 0,02 & 29,54 & $-0,56$ & 1,01 & $0,08^{*}$ \\
\hline Altura $(\mathrm{cm})$ & 26,36 & 26,45 & 74,03 & 32,63 & $-0,35$ & $-0,19$ & $0,07 * *$ \\
\hline SPAD & 8,40 & 8,20 & 6,10 & 29,41 & 0,26 & 1,08 & $0,08 * *$ \\
\hline IP & 1,52 & 2,00 & 0,84 & 60,05 & $-1,01$ & $-8,07$ & $0,02 *$ \\
\hline
\end{tabular}

${ }^{(1)} \mathrm{KS}$ : teste de normalidade de Kolmogorov-Smirvov, $\left({ }^{*}\right)$ : não significativo a 5\%, $(* *)$ : significativo a 5\%. MO: matéria orgânica; $\mathrm{SB}$ : soma de base; $\mathrm{CTC}_{\mathrm{pH}}$ : capacidade de troca de cátions a $\mathrm{pH} 7 ; \mathrm{CTC}_{\mathrm{e}}$ : capacidade de troca de cátions efetiva; V: saturação por base; m: saturação por alumínio; Ds: densidade do solo; MST: massa seca total; SPAD: valor spad; IP: intensidade de pastejo. 
baixo e que o $\mathrm{V}$ seja superior a $50 \%$. Os atributos $\mathrm{K}, \mathrm{Al}, \mathrm{m} \%$, IP apresentaram alta variabilidade e, as demais, média variabilidade. Souza et al. (2006), em trabalho com Latossolo em diferentes relevos, encontraram baixo CV para $\mathrm{pH}, \mathrm{V} \%$ e Ds. Souza et al. (2008) avaliando a variabilidade química de Argissolo em área de pastagem de Urochloa decumbens, observaram baixo $\mathrm{CV}$ para $\mathrm{pH}$ e alto para os atributos $\mathrm{Al} \mathrm{e} \mathrm{m} \%$. Altos valores de $\mathrm{CV}$ para os atributos químicos e intensidade de pastejo podem estar relacionados à heterogeneidade do manejo dos animais, à correção e à adubação empregada na área. Segundo Cavalcante et al. (2007), altos valores de CV em atributos químicos do solo ocorrem devido aos efeitos residuais de adubações anteriores, à correção da acidez do solo e à deposição dos restos culturais, o que torna a área não homogênea.

Os resultados referentes ao teste de normalidade de Kolmogorov-Smirnov indicaram que as variáveis $\mathrm{MO}, \mathrm{Ca}, \mathrm{Mg}, \mathrm{H}+\mathrm{Al}, \mathrm{CTC}_{\mathrm{pH} 7}, \mathrm{~V}$ e Ds possuem distribuição normal, que está relacionado aos valores de média e mediana próximos, bem como assimetria não acentuada (LIMA et al., 2014), essa distribuição facilita o ajuste dos semivariogramas (MACHADO et al., 2007). Silva Neto et al. (2011), em estudo da variabilidade espacial de atributos químicos do solo observaram distribuição normal apenas $\mathrm{MO}, \mathrm{CTC}_{\mathrm{pH} 7}$ e $\mathrm{CTC}_{\mathrm{e}}$, levantando a hipótese de que a falta de normalidade dos dados ocorreu devido à aplicação não uniforme de adubos ou mesmo pelo uso e manejo das áreas. Quanto às características relacionadas ao pasto, as variáveis altura e valor SPAD apresentaram distribuição normal, enquanto massa de forragem (MST) e intensidade de pastejo (IP) não foi significativo, indicando que os fatores que causam a variabilidade nas características do solo também interferem nas características relacionadas ao pasto, influenciando, em parte, o pastejo animal.

A distribuição não normal de algumas variáveis $\left(\mathrm{pH}, \mathrm{P}, \mathrm{K}, \mathrm{Al}, \mathrm{SB}, \mathrm{CTC}_{\mathrm{e}}, \mathrm{m}, \mathrm{MST} \mathrm{e}\right.$ IP) não comprometeu o estudo da área, visto que não ocorreu efeito proporcional, situação em que a média e a variabilidade dos dados são constantes, e os semivariogramas apresentam-se com patamares indefinidos e caudas alongadas, capaz de comprometer severamente as estimativas da krigagem ordinária (ISAAKS e SRIVASTAVA, 1989).

Todas as variáveis analisadas apresentaram dependência espacial (Quadro 2), a matéria orgânica (MO), densidade do solo (Ds) e a massa seca total (MST) apresentaram forte grau de dependência espacial, quanto maior o grau de dependência espacial da variável, melhor será a estimativa pela técnica da krigagem para locais não amostrados (LIMA et al., 2010). Enquanto as demais variáveis apresentaram dependência espacial moderada, resultados semelhantes aos de Souza et al., (2008) estudando atributos do solo em pastagem, apresentaram dependência espacial moderada para os atributos $\mathrm{K}, \mathrm{Ca}, \mathrm{Mg}$, $\mathrm{Al}, \mathrm{SB}, \mathrm{CTC}_{\mathrm{pH} 7}, \mathrm{CTC}_{\mathrm{e}}, \mathrm{V} \%, \mathrm{~m} \%$. A dependência espacial moderada em atributos químicos do solo pode estar relacionada ao manejo do solo, pela aplicação de adubos e corretivos, que torna o solo homogêneo em diferentes sistemas de uso e manejo (CAVALCANTE et al., 2007).

Os atributos do solo, massa seca, altura, valor SPAD e intensidade de pastejo, ajustaramse ao modelo exponencial, com coeficiente de determinação $\left(\mathrm{R}^{2}\right)$ dos modelos ajustados acima de 0,30 , com baixa instabilidade, exceto o valor SPAD com 0,16 , considerado mediamente instável. Os modelos esférico e exponencial são os mais comuns aos atributos do solo (CAMBARDELLA et al., 1994; LIMA et al., 2014 e NEVES NETO et al., 2013) e são os mais indicados para trabalhos com manejo de pastagens (PAULA NETO et al., 2014).

Os maiores valores de alcance foram de $\mathrm{Ke}$ SB com 3032 e 2586 m, respectivamente. Enquanto SPAD, MO, Mg apresentaram os menores valores com 35,41 e $44 \mathrm{~m}$, respectivamente. Os altos valores de alcance que variam entre 1198 a 3033 $\mathrm{m}$, indicam maior continuidade espacial nesses teores, independente do manejo do solo adotado. Para Corá et al. (2004), o manejo do solo realizado na área ao longo do tempo, com aplicações de fertilizantes, calcário, preparo convencional do solo, pode contribuir para maior homogeneização da área e, consequentemente, para aumento do alcance de dependência espacial de atributos do solo.

\section{REVENG}


Quadro 2. Modelos e parâmetros estimados dos semivariogramas ajustados aos valores das variáveis analisadas em área com capim-Marandu estabelecido em Argissolo Vermelho Distroférrico em Araguaína, TO (2015)

\begin{tabular}{lcccccccc}
\hline \multirow{2}{*}{ Variável } & \multicolumn{7}{c}{ Parâmetro } \\
\cline { 2 - 9 } & Modelo & $\mathbf{C}_{\mathbf{0}}{ }^{\mathbf{1}}$ & $\mathbf{C}_{\mathbf{0}}+\mathbf{C}_{\mathbf{1}}{ }^{\mathbf{2}}$ & $\mathbf{A}^{\mathbf{3}} \mathbf{( \mathbf { m } )}$ & $\mathbf{G D E}^{\mathbf{4}} \mathbf{( \% )}$ & Classe & $\mathbf{R}^{\mathbf{2 *}}$ & $\mathbf{S Q R}^{\mathbf{5}}$ \\
\hline $\mathrm{MO}$ & Exponencial & 3,80 & 15,54 & 41,00 & 75,50 & Forte & 0,50 & 97,80 \\
$\mathrm{pH}$ & Exponencial & 0,03 & 0,05 & 253,00 & 50,10 & Moderada & 0,52 & $3,44 \times 10^{-4}$ \\
$\mathrm{P}$ & Exponencial & 0,01 & 0,02 & 174,00 & 50,10 & Moderada & 0,63 & $1,17 \times 10^{-8}$ \\
$\mathrm{~K}^{+}$ & Exponencial & 0,01 & 0,02 & 3032,00 & 73,20 & Moderada & 0,80 & $7,11 \times 10^{-6}$ \\
$\mathrm{Ca}^{2+}$ & Exponencial & 0,93 & 1,87 & 315,00 & 50,00 & Moderada & 0,83 & 0,06 \\
$\mathrm{Mg}^{2+}$ & Exponencial & 0,28 & 0,55 & 44,00 & 49,20 & Moderada & 0,30 & 0,02 \\
$\mathrm{Al}^{3+}$ & Exponencial & 0,01 & 0,02 & 391,00 & 50,30 & Moderada & 0,50 & $5,16 \times 10^{-5}$ \\
$\mathrm{H}^{+} \mathrm{Al}$ & Exponencial & 0,72 & 1,45 & 1521,00 & 50,00 & Moderada & 0,71 & 0,075 \\
$\mathrm{SB}$ & Exponencial & 1,50 & 3,00 & 2586,00 & 50,00 & Moderada & 0,79 & 0,10 \\
$\mathrm{CTC}$ & Exponencial & 1,51 & 3,03 & 173,00 & 50,00 & Moderada & 0,54 & 0,37 \\
$\mathrm{CTC}$ & Exponencial & 0,94 & 1,90 & 174,00 & 50,50 & Moderada & 0,60 & 0,17 \\
$\mathrm{~V} \mathrm{( \% )}$ & Exponencial & 23,16 & 47,89 & 1198,00 & 51,60 & Moderada & 0,84 & 35,50 \\
$\mathrm{~m}(\%)$ & Exponencial & 1,58 & 3,40 & 370,00 & 53,50 & Moderada & 0,65 & 1,63 \\
$\mathrm{Ds}$ & Exponencial & 0,01 & 0,02 & 65,00 & 78,00 & Forte & 0,61 & $5,57 \times 10^{-5}$ \\
$\mathrm{MST}$ & Exponencial & 0,01 & 0,02 & 85,00 & 75,80 & Forte & 0,47 & $7,68 \times 10^{-5}$ \\
$\mathrm{Altura}$ & Exponencial & 33,70 & 67,41 & 115,00 & 50,00 & Moderada & 0,45 & 256,00 \\
$\mathrm{SPAD}$ & Exponencial & 2,80 & 6,45 & 35,00 & 56,60 & Moderada & 0,16 & 22,60 \\
$\mathrm{IP}$ & Exponencial & 0,57 & 0,86 & 786,00 & 33,50 & Moderada & 0,60 & 0,06 \\
\hline
\end{tabular}

${ }^{(1)} \mathrm{C}_{0}$ : efeito pepita; ${ }^{(2)} \mathrm{C}_{0}+\mathrm{C}$ : patamar; ${ }^{(3)} \mathrm{A}$ : alcance; ${ }^{(4)} \mathrm{GDE}$ : grau de dependência espacial; ${ }^{(5)} \mathrm{SQR}$ : soma do quadrado dos resíduos; (*) $\mathrm{R}^{2}$ : coeficiente de determinação; M.O.: matéria orgânica; SB: soma de base; $\mathrm{CTC}_{\mathrm{pH}}$ : capacidade de troca de cátions a $\mathrm{pH} 7$; $\mathrm{CTC}_{\mathrm{e}}$ : capacidade de troca de cátions efetiva; V: saturação por base; m: saturação por alumínio; Ds: densidade do solo; MST: massa seca total; SPAD: valor spad; IP: intensidade de pastejo.

Os semivariogramas ajustados para cada variável estudada constata a dependência espacial, que estima os valores não amostrados por meio do método de interpolação geoestatístico de Krigagem ordinária. Desta forma, com os valores estimados, foi possível construir mapas de isolinhas para expressar a variabilidade das variáveis avaliadas na área de estudo (Figuras 1, 2 e 3). Segundo Corá e Beraldo (2006), os mapas de isolinhas elaborados por meio da krigagem ordinária levam em consideração a dependência espacial dos atributos, possibilitando a estimativa dos valores em qualquer posição dentro do campo de estudo sem tendência e com variância mínima, proporcionando maior precisão na elaboração dos mapas.

A distribuição espacial dos atributos químicos do solo (Figuras 1 e 2) possibilita identificar regiões heterogêneas, que está relacionada ao manejo extensivo da área, os animais definem os locais preferenciais de pastejo, como cocho, aguada, sombra e disponibilidade de forragem, o que causa irregularidade na intensidade de pastejo, assim como distribuição de excretas na pastagem. Essa caracterização permite a aplicação de corretivos e fertilizantes, bem como o manejo adequado para melhor aproveitamento da área de pastagem. É possível observar grande amplitude em alguns atributos do solo. Segundo Souza et al. (2008), a 

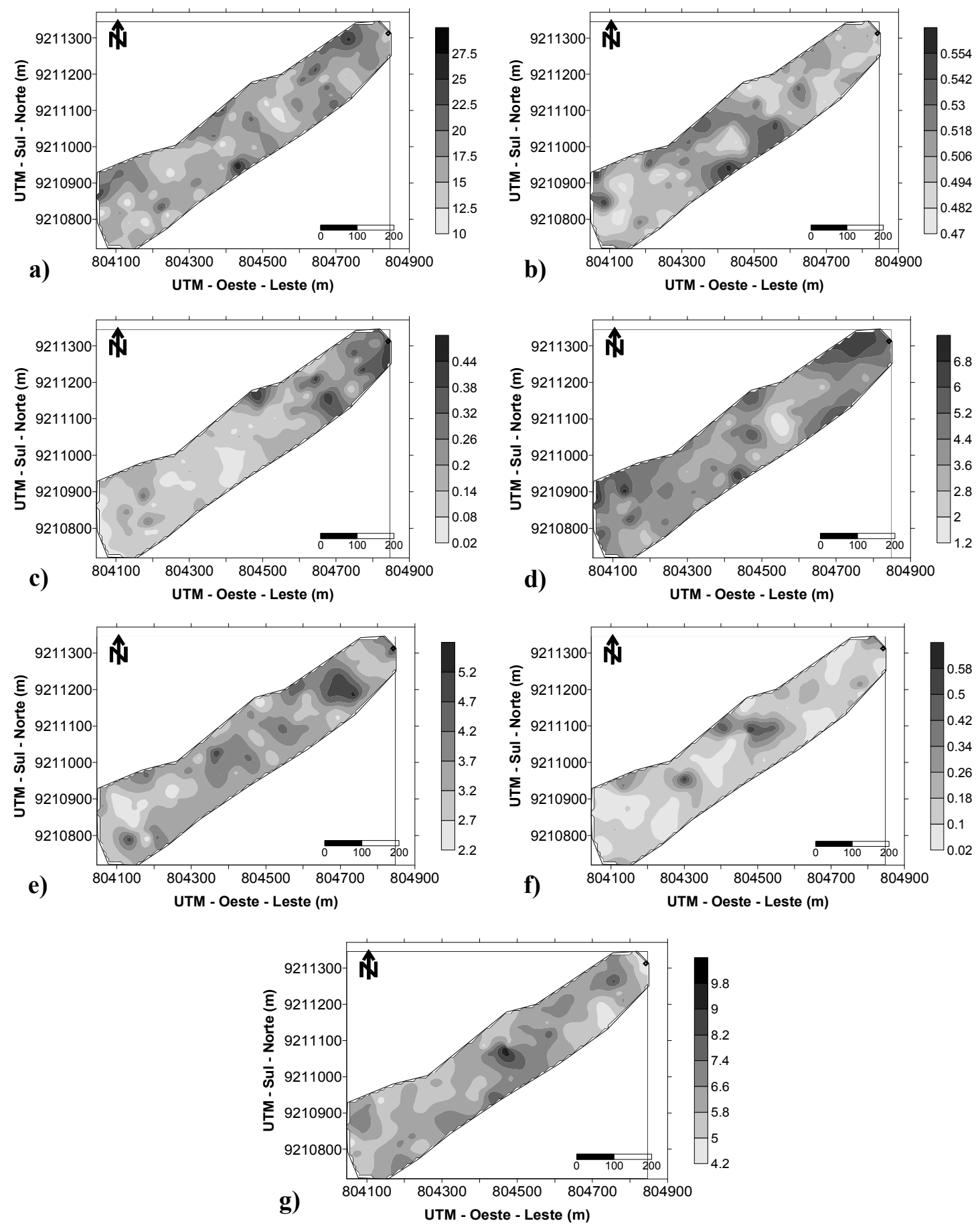

Figura 1. Mapas de isolinhas da distribuição espacial dos atributos químicos do solo: (a) MO - matéria orgânica $\left(\mathrm{g} \mathrm{kg}^{-1}\right)$; (b) $\mathrm{P}\left(\mathrm{mg} \mathrm{dm}^{-3}\right)$; (c) $\mathrm{K}^{+}\left(\mathrm{cmol}_{\mathrm{c}} \mathrm{dm}^{-3}\right)$; (d) $\mathrm{Ca}^{2+}\left(\mathrm{cmol}_{\mathrm{c}} \mathrm{dm}^{-3}\right)$; (e) $\mathrm{Mg}^{2+}\left(\mathrm{cmol}_{\mathrm{c}} \mathrm{dm}^{-}\right.$ $\left.{ }^{3}\right)$; (f) $\mathrm{Al}^{3+}\left(\mathrm{cmol}_{\mathrm{c}} \mathrm{dm}^{-3}\right)$ e (g) $\mathrm{H}+\mathrm{Al}\left(\mathrm{cmol}_{\mathrm{c}} \mathrm{dm}^{-3}\right)$. Araguaína, TO (2015).

variação nos atributos químicos revela possíveis problemas que podem ocorrer quando se usa a média dos valores para o manejo da fertilidade, pois não permite a visualização da distribuição espacial.
Por meio dos mapas de isolinhas foi possível identificar os locais de preferência de pastejo (Figura 3e) (áreas com coloração mais escuras), que também foram as áreas que apresentaram os menores valores para MST e altura (áreas com

\section{REVENG}



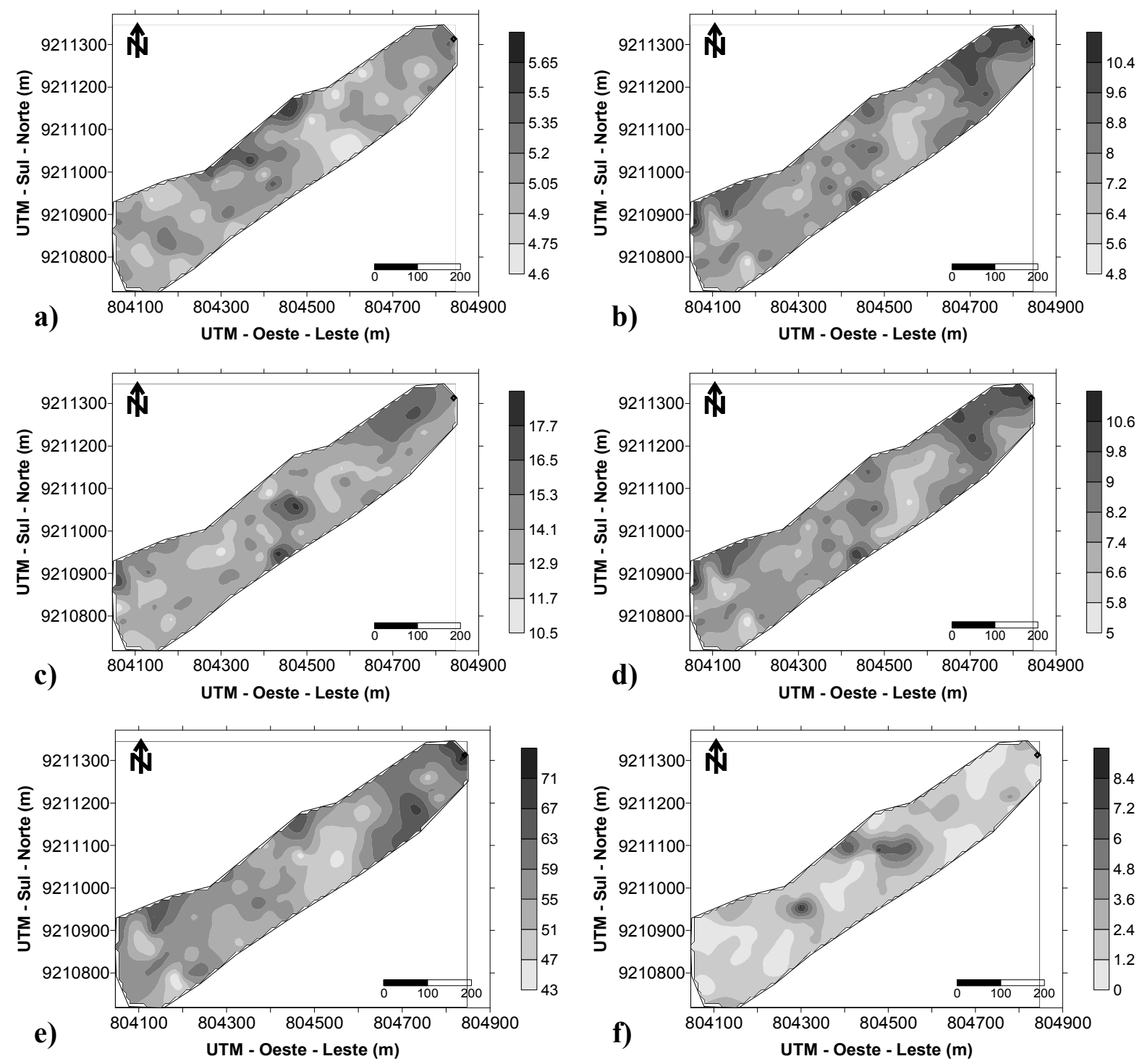

Figura 2. Mapas de isolinhas da distribuição espacial dos parâmetros: (a) $\mathrm{pH}\left(\mathrm{CaCl}_{2}\right)$; (b) $\mathrm{SB}$ - soma de base $\left(\mathrm{cmol}_{\mathrm{c}} \mathrm{dm}^{-3}\right)$; (c) $\mathrm{CTC}_{\mathrm{pH} 7}\left(\mathrm{cmol}_{\mathrm{c}} \mathrm{dm}^{-3}\right)$; (d) $\mathrm{CTC}_{\mathrm{e}}\left(\mathrm{cmol}_{\mathrm{c}} \mathrm{dm}^{-3}\right)$; (e) $\mathrm{V} \%$ - saturação por base e (f) m\% - saturação por alumínio. CTC - capacidade de troca catiônica. Araguaína, TO (2015).

coloração mais clara). Fica evidente a relação inversa da intensidade de pastejo (Figura 3e) com MST (Figura 3b) e altura do pasto (Figura 3c), indicando as zonas de preferência de pastejo na área da pastagem, que pode ter contribuído para maior densidade do solo (Figura 3a) em determinados locais, dessa forma quanto maior a altura do dossel, maior será a disponibilidade de massa de forragem, que possibilita a seleção aos animais no decorrer do pastejo (CARVALHO et al., 2009; PAULA NETO et al., 2014). Segundo Thurow et al. (2009), as pastagens heterogêneas apresentam efeito sobre a oferta de forragem, modificando a estrutura do pasto, e promovendo alterações na proporção de área ocupada pelos animais.

A distribuição do valor SPAD não variou em intensidade na área de estudo, atribui-se que a variação nos pontos preferenciais de pastejo se deve em grande parte à estrutura do pasto e aos pontos de preferência que em conjunto determinam heterogeneidade no horizonte de pastejo de pastos manejados em lotação contínua. A distribuição 

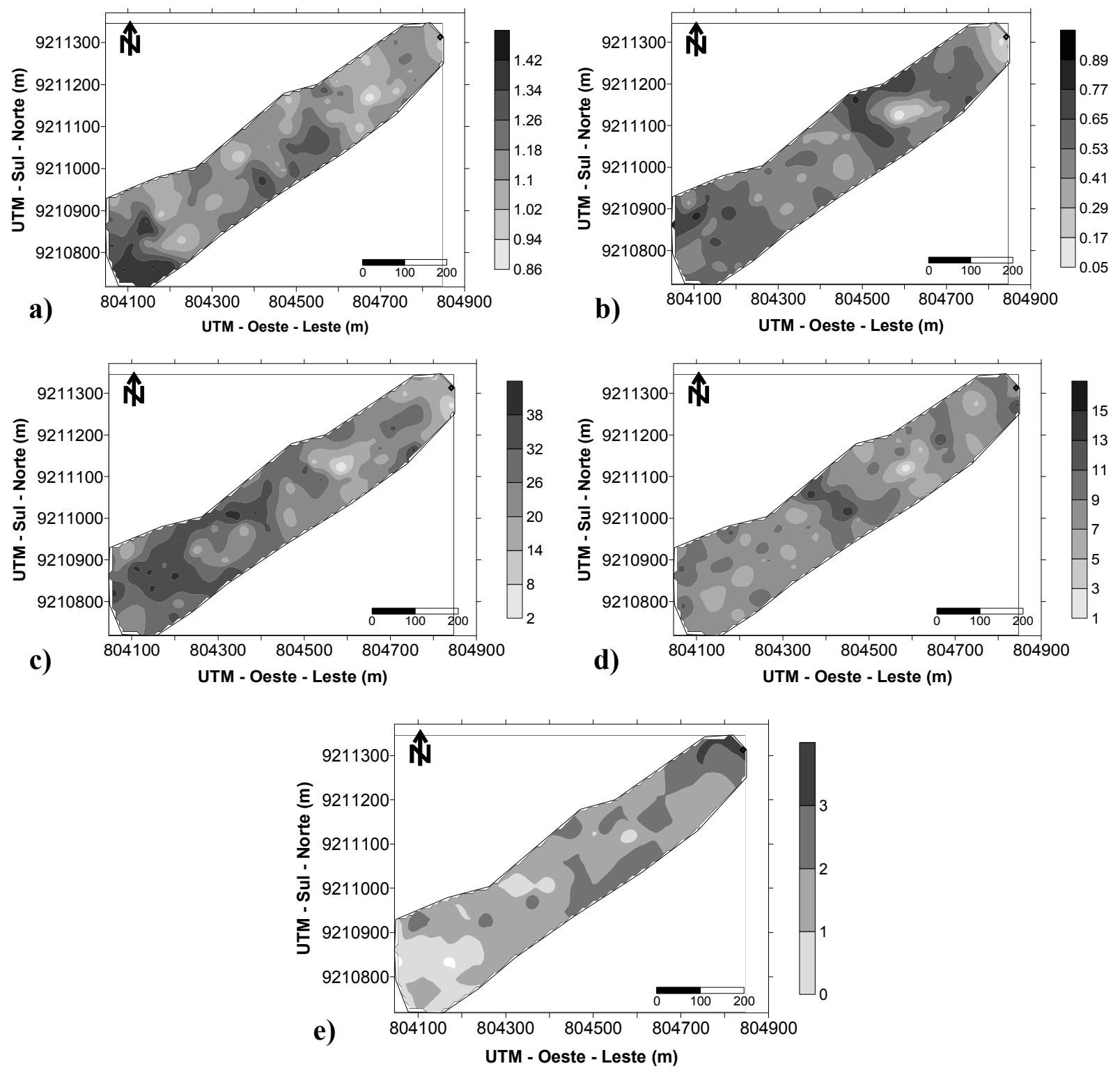

Figura 3. Mapas de isolinhas da distribuição espacial dos parâmetros: (a) Densidade do solo $\left(\mathrm{kg} \mathrm{dm}^{-3}\right)$; (b) MST ( $\left.\mathrm{kg} \mathrm{m}^{-2}\right)$; (c) Altura (cm); (d) SPAD e (e) Intensidade de pastejo. Araguaína, TO (2014).

espacial heterogênea dos atributos do solo, bem como produção de forragem e intensidade de pastejo, está relacionada ao tamanho excessivo da área da pastagem e a distribuição irregular dos pontos de preferência dos animais (aguada, cocho de sal e disponibilidade de sombra), o que causa irregularidade na intensidade de pastejo. Essa caracterização permite a aplicação de corretivos e fertilizantes, bem como o manejo do pastejo adequado para melhor aproveitamento da área de pastagem.

\section{CONCLUSÕES}

- Existe variabilidade espacial dos atributos químicos do solo que por sua vez está relacionada à maior intensidade de pastejo, menor massa seca total e altura do pasto nas zonas de preferência de pastejo em área da pastagem de Capim-Marandu, que pode ter favorecido o aumento da densidade do solo nesses locais.

- O tamanho excessivo da área e a distribuição

\section{REVENG}


irregular de cocho e aguada, resulta em regiões heterogêneas, que estão relacionados com irregularidade na intensidade de pastejo, e os mapas de isolinhas permitem estabelecer critérios de uso e manejo, com a aplicação de corretivos e fertilizantes, além do manejo do pasto para melhor aproveitamento da área.

\section{AGRADECIMENTOS}

Ao CNPq pelo apoio com as Bolsas no País/ Iniciação Científica - Edital MCT/CNPq n. ${ }^{\circ}$ 12/2010-IC, b) Edital MCT/CNPq 10/2010-Apoio Técnico/Edital MCT/CNPq 10/2010 - AT- NS (Nível Superior); e c) Bolsas no País/Produtividade em Pesquisa - PQ - 2013 - Orientador (COAGR/ CGAPB/DABS). Ao Programa de Apoio a Núcleos de Excelência - PRONEX/SECT/CNPq (Edital SECT/CNPq No 08/2010 - PRONEX). A capes pelas bolsas concedidas.

\section{REFERÊNCIAS BIBLIOGRÁFICAS}

CAMBARDELLA, C.A.; MOORMAN, T.B.; NOVAK, J.M.; PARKIN, T.B.; KARLEN, D.L.; TURCO, R.F.; KONOPKA, A.E. Field-scale variability of soil properties in Central Iowa Soils. Soil Science Society of America Journal, Madison, v.58, n.5, p.1501-1511, 1994.

CARVALHO, P.C.F.; TRINDADE, J.K.; MEZZALIRA, J.C.; POLI, C.H.E.C. NABINGER, C. GENRO, T.C.M.; GONDA, H.L. Do bocado ao pastoreio de precisão: compreendendo a interface planta-animal para explorar a multi-funcionalidade das pastagens. Revista Brasileira de Zootecnia, Viçosa, v. 38, n.spe, p.109-122, 2009.

CAVALCANTE, E.G.S.; ALVES, M.C.; SOUZA, Z.M.; PEREIRA, G.T. Variabilidade espacial de atributos químicos do solo sob diferentes usos e manejos. Revista Brasileira de Ciência do Solo, Viçosa, v.31, n.6, p.1329-1339, 2007.

CORÁ, J.E.; ARAUJO, A.V.; PEREIRA, G.T.; BERALDO, J.M.G. Variabilidade espacial de atributos do solo para adoção do sistema de agricultura de precisão na cultura de cana-de- açúcar. Revista Brasileira de Ciência do Solo, Viçosa, v.28, n.6, p.1013-1021, 2004.

CORÁ, J.E.; BERALDO, J.M.G. Variabilidade espacial de atributos do solo antes e após calagem e fosfatagem em doses variadas na cultura de canade-açúcar. Engenharia Agrícola, Jaboticabal, v.26, n.2, p.374-387, 2006.

EMBRAPA. Empresa Brasileira de Pesquisa Agropecuária. Sistema brasileiro de classificação de solos. 3. ed. Brasília: Embrapa, 2013, 353p.

EMBRAPA. Empresa Brasileira de Pesquisa Agropecuária. Manual de análises químicas de solos, plantas e fertilizantes. 2 ed. Brasília: Embrapa Produção de Informação, 2009. 627p.

EMBRAPA. Empresa Brasileira de Pesquisa Agropecuária. Manual de métodos de análises de solo.2.ed. Rio de Janeiro: Centro Nacional de Pesquisa de Solos, 1997. 212p.

GOLDEN SOFTWARE. Surfer for Windows version 8.0. Colorado: Golden, 2002.

ISAAKS, E.H.; SRIVASTAVA, R.M. An introduction to applied geoestatistics. New York: Oxford University, 1989. 561 p.

KÖEPPEN, W. Climatologia: conunestudio de los climas de laTierra. México: Fondo de Cultura Economica, 1948. 478p.

LIMA, G.C.; SILVA, M.L.N.; OLIVEIRA, M.S.; CURI, N.; SILVA, M.A.; OLIVEIRA, A.H. Variabilidade de atributos do solo sob pastagens e mata atlântica na escala de microbacia hidrográfica. Revista Brasileira de Engenharia Agrícola e Ambiental, Campina Grande, v.18, n.5, p.517526, 2014.

LIMA, J.S.S.; SOUZA, G.S.; SILVA, S.A. Amostragem e variabilidade espacial de atributos químicos do solo em área de vegetação natural em regeneração. Revista Árvore, Viçosa, v.34, n.3, p.127-136, 2010. 
MACEDO, M.C.M.; ZIMMER, A.H.; KICHEL, A.N.; ALMEIDA, R.G.; ARAÚJO, A. R. Degradação de pastagens, alternativas de recuperação e renovação, e formas de mitigação. Encontro de adubação de pastagens da Scot Consultoria-Tec-Fértil, Ribeirão Preto p.158181, 2013.

MACHADO, L.O.; LANA, A.M.Q.; LANA, R.M.Q.; GUIMARÃES, E.C.; FERREIRA, C.V. Variabilidade espacial de atributos químicos do solo em áreas sob sistema plantio convencional. Revista Brasileira de Ciência do Solo, Viçosa, v. 31, n.3, p.591-599, 2007.

MARCHÃO, R.L.; BECQUER, T.; BRUNET, D.; BALBINO, L.C.; VILELA, L.; BROSSARD, M. Carbon and Nitrogen stocks in Brazilian clayer Oxisol: 13-year effects of integrated crop-livestock management systems. Soil and Tillage Research, Amsterdam, v.103, n.2, p.442-450, 2009.

MELO, J.C.; ALEXANDRINO, E.; PAULANETO, J.J.; SILVA, A.A.M.; NEIVA, J.N.M.; REZENDE, J.M. Preferência de forragem de Capim-Marandu (Urochloa brizantha cv. Marandu) manejado sob lotação intermitente e submetido à doses de nitrogênio na Amazônia legal. Semina: Ciências Agrárias, Londrina, v.36, n.4, p-1679-0359, 2015.

NEVES NETO, D.N.; SANTOS, A.C.; SANTOS, P.M.; MELO, J.C.; SANTOS, J.S. Análise espacial de atributos do solo e cobertura vegetal em diferentes condições de pastagem. Revista Brasileira de Engenharia Agrícola e Ambiental, Campina Grande, v.17, n.9, p.995-1004, 2013.

PÁSCOA, A.G.; COSTA, M.J.R.P. Aplicação dos sistemas de informação geográfica para definição de estratégias de manejo de bovinos nas pastagens. Revista Brasileira de Zootecnia, Viçosa, v.36, n.spe, p.45-51, 2007.

PAULA NETO, J.J.; ALEXANDRINO, E.; SANTOS, A.C.; MENDES FILHO, G.O.; SILVA, D.P.; MELO, J.C. Distribuição espacial da altura do dossel e efeito sobre a cobertura do solo em pastos mantidos em lotação contínua. Bioscience
Journal, Uberlândia, v. 30, sup.2, p.650-658, 2014.

ROBERTSON, G.P. GS+: Geostatistics for the environmental sciences - GS+ User'sguide. Plainwell, Gamma Design Software, 2008. 152p.

SANA, R.S.; ANGHINONI, I.; BRANDÃO, Z.N.; HOLZSCHUH, M.J. Variabilidade espacial de atributos físico-químicos do solo e seus efeitos na produtividade do algodoeiro. Revista Brasileira de Engenharia Agrícola e Ambiental, Campina Grande, v.18, n.10, p.994-1002, 2014.

SILVA, D.J.; QUEIROZ, A.C. Análise de Alimentos: métodos químicos e biológicos. $2^{\circ} \mathrm{Ed}$. Viçosa: UFV, 2002. 165p.

SILVA NETO, S.P.; SANTOS, A.C.; LEITE, R.L.L.; DIM, V.P.; CRUZ, R.S.; PEDRICO, A.; NETO, D.N.N. Análise espacial de parâmetros da fertilidade do solo em região de ecótono sob diferentes usos e manejos. Semina: Ciências Agrárias, Londrina, v.32, n.2, p. 541-552, 2011.

SILVA, R.G.; NEIVA, J.N.M; SANTOS, A.C.; CÂNDIDO, M.J.D.; SOUSA, A.L.D.; LOPES, M.N.; SILVA, R.O.; CARNEIRO, L.C. Spatial distribution of feces and estimates of nitrogen return by dairy cows on mombasa grass pastures. Revista Brasileira de Zootecnia, Viçosa, v.42, n.5, p.369-380, 2013.

SOUZA, G.S.; LIMA, J.S.S.; SILVA, S.A.; OLIVEIRA, R.B. Variabilidade espacial de atributos químicos em um Argissolo sob pastagem. Acta Scientiarum Agronomy, Maringá, v.30, n.4, p.589-596, 2008.

SOUZA, Z.M.; MARQUES JUNIOR, J. PEREIRA, G.T.; MONTANARI, R. Otimização amostral de atributos de latossolos considerando aspectos solo-relevo. Ciência Rural, Santa Maria, v.36, n.3, p.829-836, 2006.

THUROW, J.M.; NABINGER, C.; CASTILHOS, Z.M.S.; CARVALHO, P.C.F.; MEDEIROS, C.M.O.; MACHADO, M.D. Estrutura da vegetação e comportamento ingestivo de novilhos e pastagem 
natural do Rio Grande do Sul. Revista Brasileira de Zootecnia, Viçosa, v.38, n.5, p.818-826, 2009.

TREVISAN, N.B; QUADROS, F.L.F; SILVA, A.C.F; BANDINELLI, D.G; MARTINS, C.E.N. Efeito da Estrutura de uma Pastagem Hibernal sobre o Comportamento de Pastejo de Novilhos de Corte. Revista Brasileira de Zootecnia, Viçosa, v.34, n.3, p.774-780, 2005.

VIEIRA, S.R. Geoestatística em estudos de variabilidade espacial do solo. In: NOVAIS, R.F. de; ALVAREZ V., V.H.; SCHAEFER, C.E.G.R. (Ed.). Tópicos em ciência do solo. Viçosa: Sociedade Brasileira de Ciência do solo, 2000. p.154.

WARRICK, A.W.; NIELSEN, D.R. Spatial variability of soil physical properties in the field. In: HILLEL, D. (Ed.). Application of soil physics. New York: Academic Press, 1980. 